This material is posted here with permission of the IEEE. Such permission of the IEEE does not in any way imply IEEE endorsement of any of Helsinki University of Technology's products or services. Internal or personal use of this material is permitted. However, permission to reprint/republish this material for advertising or promotional purposes or for creating new collective works for resale or redistribution must be obtained from the IEEE by writing to pubs-permissions@ieee.org.

By choosing to view this document, you agree to all provisions of the copyright laws protecting it. 


\section{Semiblind Source Separation of Climate Data Detects El Niño as the Component with the Highest Interannual Variability}

\author{
Alexander Ilin \\ Neural Networks Research Centre \\ Helsinki University of Technology \\ P.O. Box 5400, FI-02015 TKK, \\ Espoo, Finland \\ E-mail: Alexander.Ilin@tkk.fi
}

\author{
Harri Valpola \\ Lab. of Computational Engineering \\ Helsinki University of Technology \\ P.O. Box 9203, FI-02015 TKK, \\ Espoo, Finland \\ E-mail: Harri.Valpola@tkk.fi
}

\author{
Erkki Oja \\ Neural Networks Research Centre \\ Helsinki University of Technology \\ P.O. Box 5400, FI-02015 TKK, \\ Espoo, Finland \\ E-mail: Erkki.Oja@tkk.fi
}

\begin{abstract}
Denoising source separation (DSS), a recently developed source separation framework, was applied to extracting components exhibiting slow, interannual temporal behaviour from climate data. Three datasets with daily measurements were used: surface temperature, sea level pressure and precipitation around the globe. For all datasets, the first extracted component captured the well-known El Niño-Southern Oscillation phenomenon and the second component was close to the derivative of the first one. Several other components with slow dynamics were extracted and together the components appear to capture essential features of the slow-dynamics state of the climate system. The first two components were identified reliably but the following components may have remained mixed. Nonlinear DSS could identify the physically most meaningful rotation among them but only linear DSS was within the scope of this paper. This paper offers a simple demonstration of exploratory data analysis of climate data by DSS and suggests future lines of research.
\end{abstract}

\section{INTRODUCTION}

El Niño-Southern Oscillation (ENSO) is a global-scale phenomenon in the ocean and atmosphere, known as one of the most prominent sources of interannual variability in weather and climate around the world. Its oceanic component (El Niño-La Niña events) can be defined as a Pacific basin-wide increase (El Niño) or decrease (La Niña) in the sea surface temperatures in the central and/or eastern Pacific Ocean [1]. The warm El Niño events are known to be accompanied by the decrease in the sea level atmospheric pressure in the western Pacific, which is the atmospheric component of ENSO called Southern Oscillation (SO). SO can be defined as a large-scale oscillation of the air mass between the southeastern tropical Pacific and the Australian-Indonesian regions.

The intensity of ENSO events is traditionally quantified using simple indices calculated from monthly averaged measurements taken in special locations. Usually the analysis is applied to so-called anomalies which refer to deviations from average seasonal variation. For example, the intensity of El Niño can be estimated using the sea surface temperature (SST) anomalies averaged over the Niño 3 region $\left(5^{\circ} \mathrm{N}-\right.$ $\left.5^{\circ} \mathrm{S}, 150^{\circ}-90^{\circ} \mathrm{W}\right)[2],[3]$, and the most commonly used
SO index (SOI) is computed from monthly mean sea level pressure anomalies measured at Tahiti $\left(17.5^{\circ} \mathrm{S}, 149.6^{\circ} \mathrm{W}\right)$ and Darwin $\left(12.4^{\circ} \mathrm{S}, 130.9^{\circ} \mathrm{E}\right)$, in Australia. The spatial locations included in the simple ENSO indices are chosen such that their teleconnectivity properties are maximized [4] but their choice is still somewhat arbitrary.

The global ENSO patterns are usually computed as correlations of local anomalies in surface temperature, sea level pressure or other variables with a chosen ENSO index or using regression coefficients from the anomalies to that index [3]. The obtained spatial patterns depend on the choice of the used ENSO indices. For example, the sea level pressure correlation patterns naturally have peaks over Darwin and Tahiti.

Another possibility to capture ENSO events and describe their global patterns is applying empirical orthogonal function (EOF) analysis to pre-filtered anomaly data. EOF analysis is the name for principal component analysis (PCA) used in the climate literature. The first EOF maps usually represent the ENSO patterns and the first principal component is a good index of the major warm and cold events in the tropical Pacific.

In this paper, we search for physically meaningful states with slow, interannual time course from climate data. Diurnal and annual variability in solar radiation means that the climate system has daily and annual cycles but climate events such as ENSO whose time course is slower than the annual cycle are in evidence that the climate system has intrinsic interannual dynamics.

We show that the basic ENSO events (El Niño and SO) appear as the component with most prominent interannual variability from the global measurements of surface temperature, sea level pressure and precipitation. This is done using a recently developed method called denoising source separation (DSS) [5] which can be viewed as an extension of independent component analysis (ICA) [6]. The extracted ENSO-related component appears to be an important dimension of the climate state-space but the method uncovers several other components whose temporal behaviour exhibits relatively slow, interannual evolution. 


\section{MATERIALS AND METHODS}

\section{A. DSS method}

DSS is a general algorithmic framework which can be used for capturing interesting hidden phenomena from multivariate data [5]. As in PCA, factor analysis and ICA, the underlying data model in basic DSS method is linear. The modelling assumption is that there are some components (also called factors, sources or state variables depending on the context) which are reflected in the measurements through a linear mapping. In the context of factor analysis this mapping is called the loading matrix and in ICA the mixing matrix.

The goal of the analysis is to identify the unknown components and corresponding loading vectors given the data. In our case the components should correspond to the states of the climate system. Since we use spatial maps of daily recordings of weather-related variables as the data, the loading vectors are spatial "weather maps" which show the typical weather pattern that the extracted state variables correspond to.

The charasteristic feature of DSS algorithms is that the definition of "interesting phenomenon" comes in the form of a denoising procedure. DSS can be linear or nonlinear depending on whether the denoising is linear or nonlinear. In both cases the first step of the analysis is so-called whitening or sphering. It amounts to normalising the data such that the covariance structure becomes uniform: unit projections of the whitened data always produce unit-variance signals. Whitening can be implemented by PCA. Since the principal components are uncorrelated, the data can be whitened simply by normalising the components to unit variance. The dimensionality of the data can also be reduced at this stage by retaining only the components corresponding to largest eigenvalues.

Whitening may seem counterproductive at first because after whitening, any useful structure that PCA could use is abolished. The benefit is that for whitened data, the loading vectors of different components can be expected to be roughly orthogonal and for this reason whitening is the first step in many ICA algorithms. Unlike in PCA but similarly to ICA, the original loading vectors are not restricted to be orthogonal which may result in more physically meaningful representations of the data.

In linear DSS, whitening is followed by a linear filtering step which renders the variance for some components higher than for others. Linear PCA can then identify these components. The eigenvalues obtained by PCA tell the variance of the extracted components but due to the initial whitening, the eigenvalue corresponds to the ratio between variance after filtering and before filtering. This is the objective function for linear DSS algorithms. The components are ranked according to the prominence of the desired properties the same way as the principal components in PCA are ranked according to the amount of variance they explain.

In the present work, we aim at finding components which exhibit prominent variability in the interannual timescale. Therefore, the whitened data were filtered using a band-pass filter whose frequency response is shown in Fig. 1.

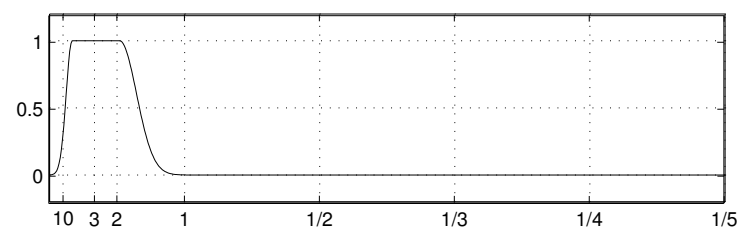

Fig. 1. Frequency response of the filter used in DSS. The abscissa is linear in frequency but is labeled in terms of periods, in years.

PCA, which is used as the last step of linear DSS, can only separate components which have distinct eigenvalues. Due to noise, finite sample size and unaccurate modelling assumptions, separation is in practice reliable only if the eigenvalues are clearly distinct. Due to the limited scope of this article, we concentrate on linear DSS although it is clear that nonlinear filtering can identify more reliably the physically most meaningful separation. In particular, nonlinear DSS can in many cases reliably separate components whose "eigenvalues" are similar. The main implementational difference between linear and nonlinear DSS is that the nonlinear filtering needs to be embedded in an iterative power-method implementation of PCA [5].

\section{B. Data and preprocessing method}

We used globally gridded measurements of major atmospheric variables over a long period of time. These data are provided by the reanalysis project of the National Centers for Environmental Prediction-National Center for Atmospheric Research (NCEP/NCAR) [7]. We applied the analysis to the same three datasets as [3]: surface temperature, sea level pressure and precipitation data coming from the NCEP/NCAR reanalysis project.

Although the quality of the data is worse for the beginning of the reanalysis period and it considerably varies throughout the globe, we used the whole period of 1948-2004. The data are daily measurements from regularly spaced locations over the globe with $2.5^{\circ} \times 2.5^{\circ}$ resolution.

The long-term mean was removed from the data and the effect of a denser sampling grid around the poles was taken into account by multiplying each data point by a weight proportional to the square root of the local area of its location. Then, we reduced the spatial dimensionality of the data using the PCA/EOF analysis applied to the weighed data. For each dataset, we retained 100 principles components which explain more than $90 \%$ of the total variance. We applied the analysis for each dataset separately and for combined data.

The comparison indices SOI and Niño 3 SST are available as a monthly timeseries. In order to be able to compare them with the results obtained by DSS, we expanded them into daily measurements and computed the projection which is analogous to the projection vector in DSS. We also computed the corresponding timeseries from the whitened data in order to be able to evaluate the objective function, the ratio between the variance after and before filtering for daily measurements. 


\section{RESULTS}

\section{A. Surface temperature}

The four most prominent interannual components extracted from the surface temperature data are shown in Fig. 2. The time course of the first component (upper curve in Fig. 2) shows striking resemblance with the El Niño index from the sea surface temperature in the Niño 3 region (lower curves). Note that the upper components are extracted from climate data consisting of daily measurements from the whole globe, with the only constraint being the emphasis on strong interannual oscillations. Also note that the values of Niño 3 SST index are monthly averages and consequently appear smoother than the daily averages of DSS components. Regressing the Niño 3 SST index from the whitened data reveals the corresponding daily timeseries (bottom of Fig. 2).

The spatial patterns corresponding to the four leading components are shown in Fig. 3. The first DSS map contains many features traditionally associated with El Niño: the strongest pattern in central and eastern tropical Pacific with broader regions along the eastern Pacific coast, a negatively correlated "boomerang"-shaped region at $20^{\circ}-40^{\circ}$ latitude in both hemispheres linked in the far western equatorial Pacific, positive values in the Indian Ocean, negative values in the North Pacific and around New Zealand [3]. Similar features are observed from the regression pattern in Fig. 4 calculated using the Niño 3 SST index.

However, some features are more distinctive on the DSS map, for example, a strong teleconnection in southern Africa, strong negative regions in the southern Atlantic and in southwestern parts of the United States. Some other features usually associated with El Niño are much weaker, for example, the dipole structure in the tropical Atlantic reported in [8].

The second component also appears to be related to El Niño and roughly corresponds to the time derivative of the first component. The third and the fourth components show distinct oscillations with a 2-3 year period. The loadings in Fig. 3 are relatively strong at the poles which is partly due to the fact that overall variability in climatic conditions is strongest at the poles.

\section{B. Sea level pressures}

The leading components with interannual variability extracted with DSS are shown at the middle columns of Figs. 2 (time course) and 3 (spatial patterns). The first component again bears striking similarity with the well-known SO phenomenon both in the time course and in the spatial pattern. The DSS map in Fig. 3 is similar to the classical SO pattern [3]: there is a major structure seesaw in the Tropics and subtropics, large pressure departures in the North Pacific, a quadrapolelike structure in the Australasia-South Pacific region with the clear Pacific-South American pattern [9]. Note that the negative center south to Australia is very weak and shifted more towards New Zealand compared to the SO patterns documented in the literature.

The classical SO structure is clearer from the regression coefficients shown in Fig. 4. Compared to it, the DSS pattern is characterized by the relatively weaker negative regions over the North and South Atlantic and the more dominant patterns in the North and South Pacific.

The three following components demonstrate oscillatory behaviour with roughly 2-3-year period. Judging from their time course, the components do not appear to be the same as the ones extracted from the surface temperature data.

\section{Precipitation}

The leading components of interannual variability for the precipitation data are shown in the rightmost column in Fig. 2 (time course) and Fig. 3 (spatial patterns). The first component again resembles the structure of the ENSO phenomenon: The dominant effects are clearly seen throughout the tropical Pacific with maximum values in the Niño 3 region. The clear patterns are the intertropical convergence zone (ITCZ) and South Pacififc convergence zone (SPZC), a "boomerang"shaped negatively correlated area in mid-latitudal Pacific merged over Indonesia, positive values in the Indian Ocean and subtropical and tropical Atlantic.

Like in the classical ENSO patterns [3], the resemblance between the maps of the first DSS components for the surface temperature and precipitation is striking (see the first row in Fig. 3): The warm areas mostly correspond to wet areas, which shows the importance of local evaporation in the ENSO.

The second DSS component is very similar to the second component extracted from the surface temperature data. The spatial pattern has an interesting localization in the Niño 3 region with positively correlated regions over Hawaii and near the Chilian coast. The rest of the tropical and subtropical areas mostly have negative loadings. The following two components again clearly demonstrate slow oscillations but they do not appear to be the same as the ones extracted from the two other datasets.

\section{Combined data}

The leading inerannual components extracted from the combined data including surface temperature, sea level pressure and precipitation are shown in Fig. 5 (time course) and Fig. 6 (spatial patterns). As before, the first extracted component is a good ENSO index. The corresponding spatial patterns shown in the first row of Fig. 6 are very similar to the maps from the first row of Fig. 3.

The second extracted component bears similarity to the second component extracted from the surface temperature and precipitation data. The most significant difference is the sea level pressure loadings which now have a clear pattern in the Pacific region. Note that the time course of this component resembles the time derivative of the ENSO index (see Fig. 5).

The strong interannual character of the third component is mostly defined by the sea level pressure and precipitation measurements: Their spatial patterns are similar to the third components extracted from the separate datasets.

The fourth component is similar to the fourth component extracted separately from the surface temperature and sea level pressure. It is again localized near the North Pole. 

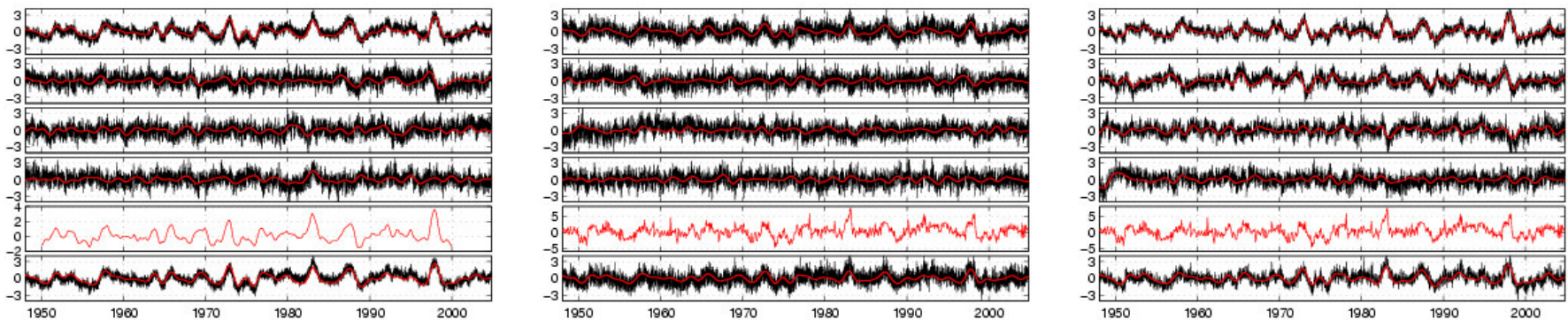

Fig. 2. Most prominent components extracted from three datasets separately. Top four: The time course of the first leading components (black) and thei filtered versions found by DSS (red). The first component at the top. The non-filtered components are normalized to unit variance. Bottom two: ENSO index (the Niño 3 SST index for surface temperature and the inverted SOI for sea level pressure and precipitation), the regressed component (black) and its filtered version (red).

Surface temperature

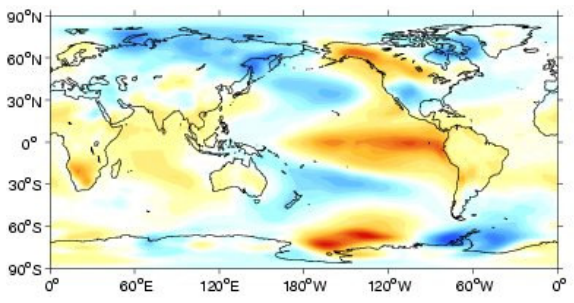

$\begin{array}{lllllllll}-0.8 & -0.6 & -0.4 & -0.2 & 0 & 0.2 & 0.4 & 0.6 & 0.8\end{array}$

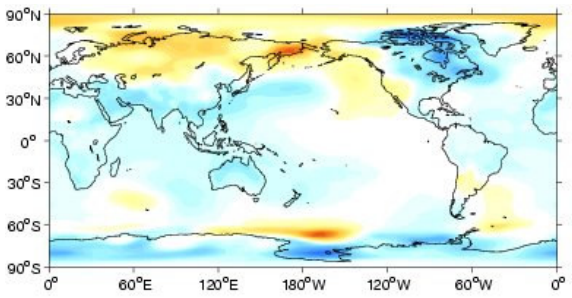

$\begin{array}{lllllllll}-0.8 & -0.6 & -0.4 & -0.2 & 0 & 0.2 & 0.4 & 0.6 & 0.8\end{array}$
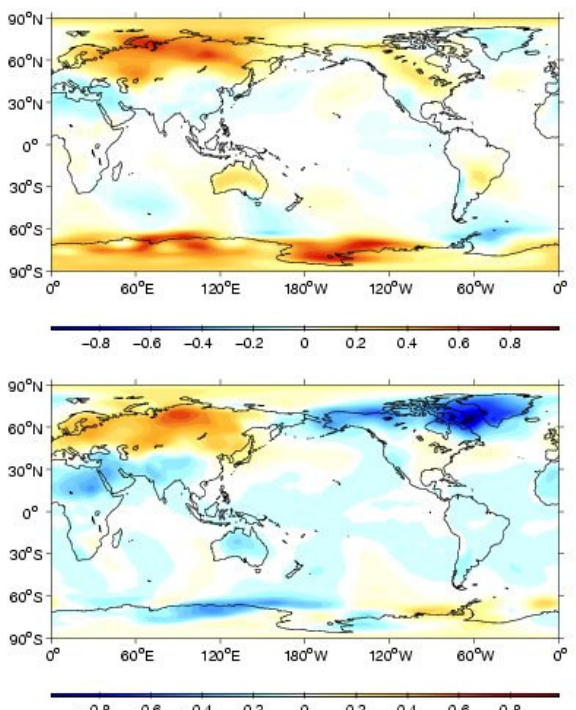

Sea level pressure
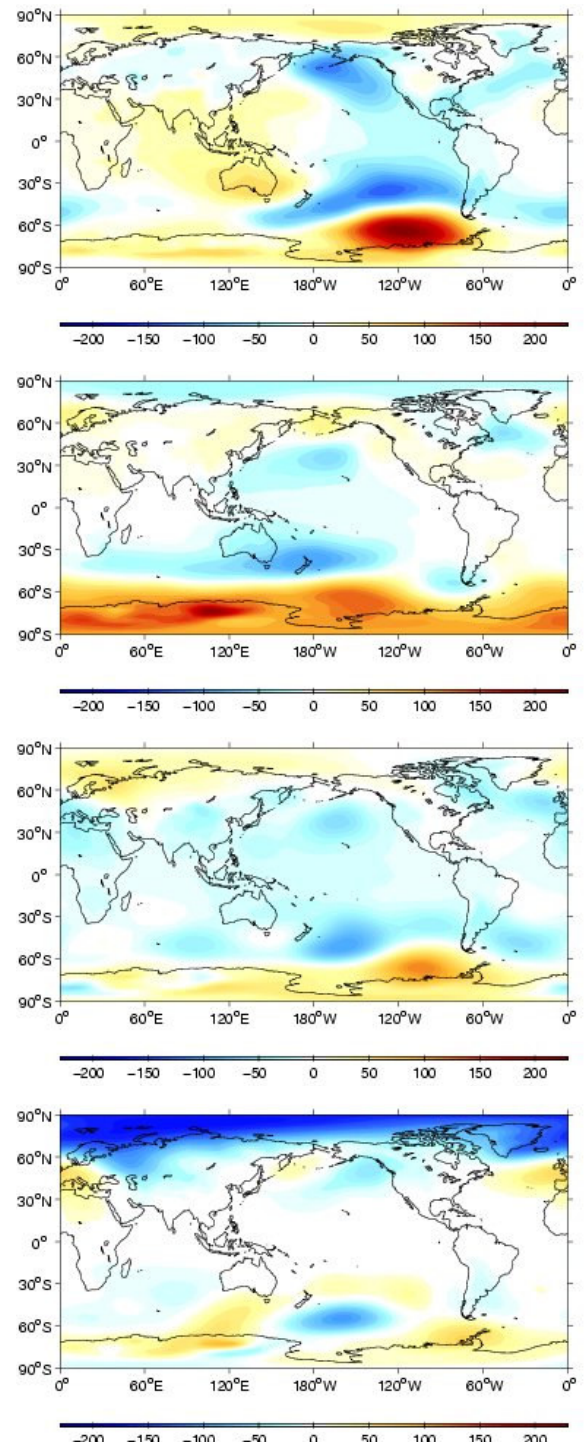

Precipitation
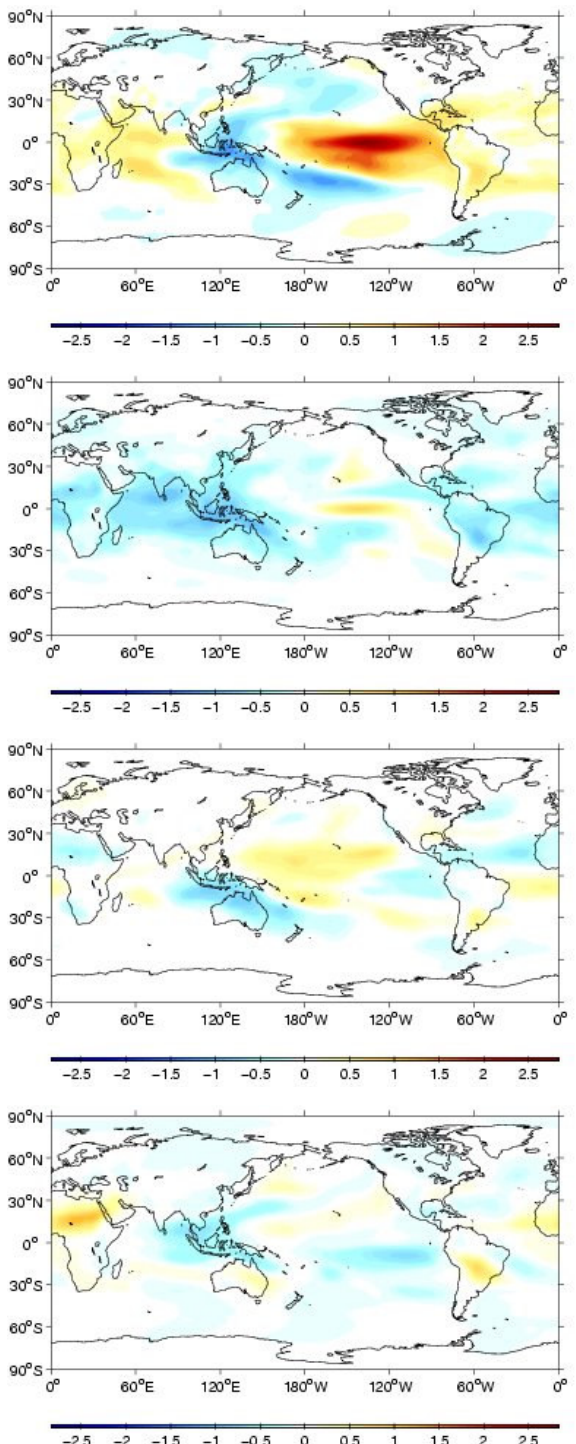

Fig. 3. The spatial patterns (loading vectors) of the four leading interannual components extracted from three datasets separately. The first component at the top. The loading vectors are normalised such that the signal of interest (timeseries after filtering) was normalised to unit variance. The maps thus tell how strongly the slowly evolving state variable is expressed in the measurement data. 
Surface temperature

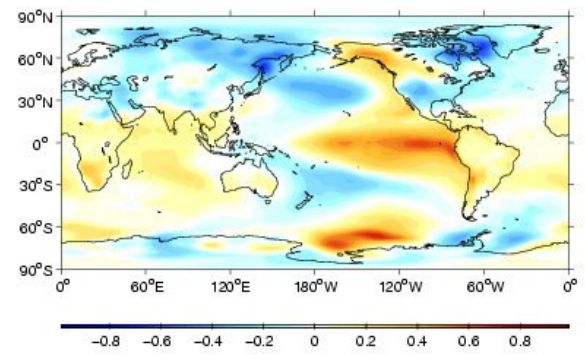

Sea level pressure

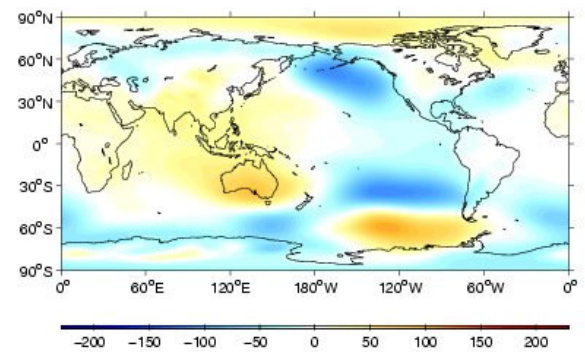

Precipitation

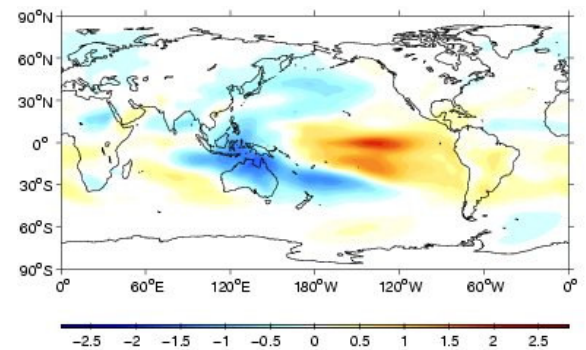

Fig. 4. The regression coefficients from the Niño 3 SST index (surface tempreature) and the inverted SOI (sea level pressure and precipitation).

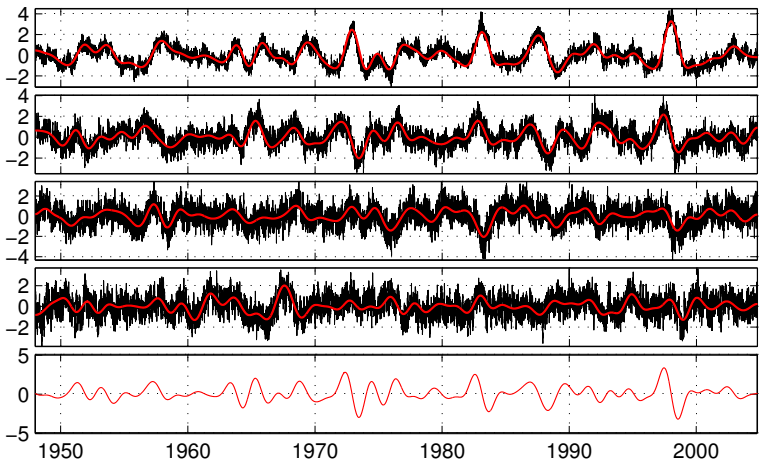

Fig. 5. Most prominent components extracted from the combined data. Top four: The time course of the first leading components (black) and their filtered versions found by DSS (red). The non-filtered components are normalized to unit varaince. Bottom: The derivative of the topmost filtered component bares similarity to the second component.

TABLE I

CORRELATIONS OF THE FIRST COMPONENT AND ENSO INDICES

\begin{tabular}{|c|cccc|}
\hline & $\begin{array}{c}\text { Surface } \\
\text { temperature }\end{array}$ & $\begin{array}{c}\text { Sea level } \\
\text { pressure }\end{array}$ & Precipitation & $\begin{array}{c}\text { Combined } \\
\text { data }\end{array}$ \\
\hline Niño 3 SST & 0.9765 & 0.9502 & 0.9513 & 0.9323 \\
SOI & 0.9417 & 0.9264 & 0.8300 & 0.8024 \\
\hline
\end{tabular}

Table I lists the correlation coefficients between the first extracted component and SOI and Niño 3 SST index. Somewhat surprisingly even the component extracted from sealevel-pressure data resembles more Niño 3 SST index than SOI although SOI is defined in terms of sea level pressure. Moreover, for combined data the correlation is weaker than for surface temperature. Table II lists the variance of the filtered components divided by the variance of the non-filtered ones. This is the index which is used as the objective function for extracting the components. The first extracted component therefore always has the largest value in all conditions.

\section{DISCUSSION}

The same first or first two components are found in all three datasets which is strong evidence in support of physical meaningfulness. In linear DSS, each component can be characterised by their eigenvalue and only components whose eigenvalues are clearly different can be reliably separated. This is analogous to PCA where components with equal eigenvalues form subspaces with rotational indeterminacy. The eigenvalues of the components extracted from the combined
TABLE II

VALUES OF THE OBJECTIVE FUNCTION

\begin{tabular}{|c|cccc|}
\hline & $\begin{array}{c}\text { Surface } \\
\text { temperature }\end{array}$ & $\begin{array}{c}\text { Sea level } \\
\text { pressure }\end{array}$ & Precipitation & $\begin{array}{c}\text { Combined } \\
\text { data }\end{array}$ \\
\hline Comp. 1 & 0.6156 & 0.2782 & 0.6182 & 0.7484 \\
Comp. 2 & 0.2031 & 0.1275 & 0.4005 & 0.5105 \\
Comp. 3 & 0.1691 & 0.1068 & 0.2126 & 0.3376 \\
Comp. 4 & 0.1604 & 0.0963 & 0.1874 & 0.3014 \\
Comp. 5 & 0.1185 & 0.0772 & 0.1343 & 0.2993 \\
Niño 3 SST & 0.5906 & 0.2561 & 0.5792 & 0.6853 \\
SOI & 0.5547 & 0.2434 & 0.4634 & 0.5354 \\
\hline
\end{tabular}

data in Table II further support the finding that the first two components can be reliably identified while the following three components form a subspace within which the physically most meaningful rotation may not have been identified. The values in Table II are good predictors of the similarity of the components in different conditions. It should also be noted that even if the best rotation of the components is not found, the subspace clearly captures physically meaningful state variables of the climate system. This is clearly evident in the prominent temporal structure present in all the extracted components.

Nonlinear filtering can separate processes which have similar eigenvalues. Moreover, as the signals of interest are state variables which have a predictable time course, an important future line of research will be to model nonlinear dynamics of the state variables and extend the denoising to be nonlinear. In the global climate system, everything depends on everything else, but a sensible criterion for separation is that the states should have as little couplings as possible (cf. this physical independence with statistical independence criterion in ICA). A similar separation criterion was used in [10].

Nonlinear effects should also be taken into account when analysing the results because they are known to exist between the state variables. The most prominent phenomenon in climate system is the seasonal variation and it is known that ENSO has different effects depending on the time of the year, that is the combined effect of ENSO and annual oscillation has a nonlinear component. Similar nonlinear effects can be expected to be present among all state variables but they are not revealed by static loading matrices.

In this paper we demonstrated the first time how DSS can be applied to exploratory data analysis of climate data. We showed that it is possible to identify patterns which reflect physically meaningful dynamics, which can be useful for both 
Surface temperature
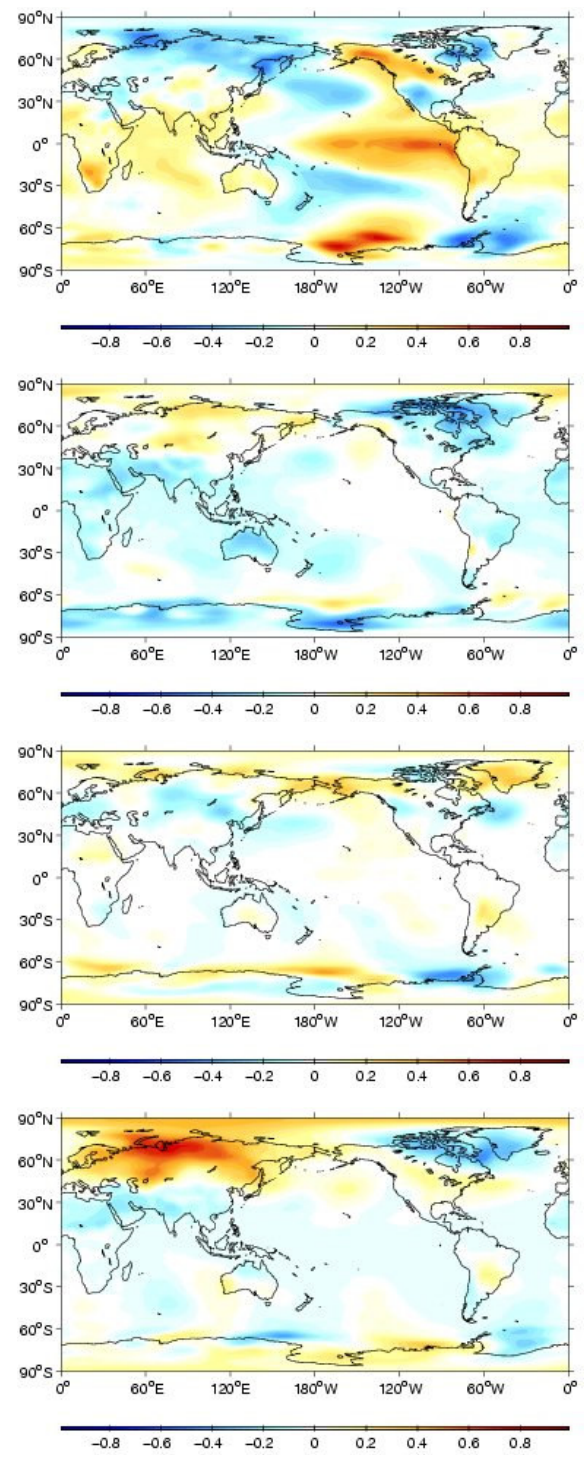

Sea level pressure
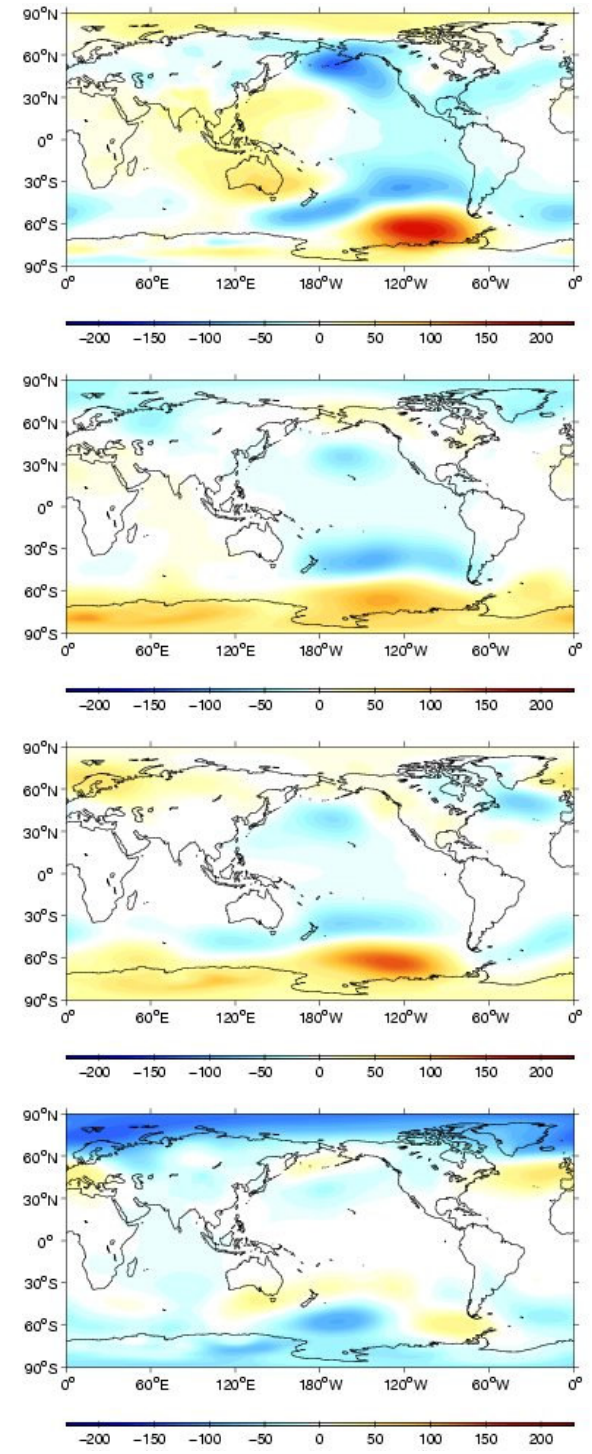

Precipitation
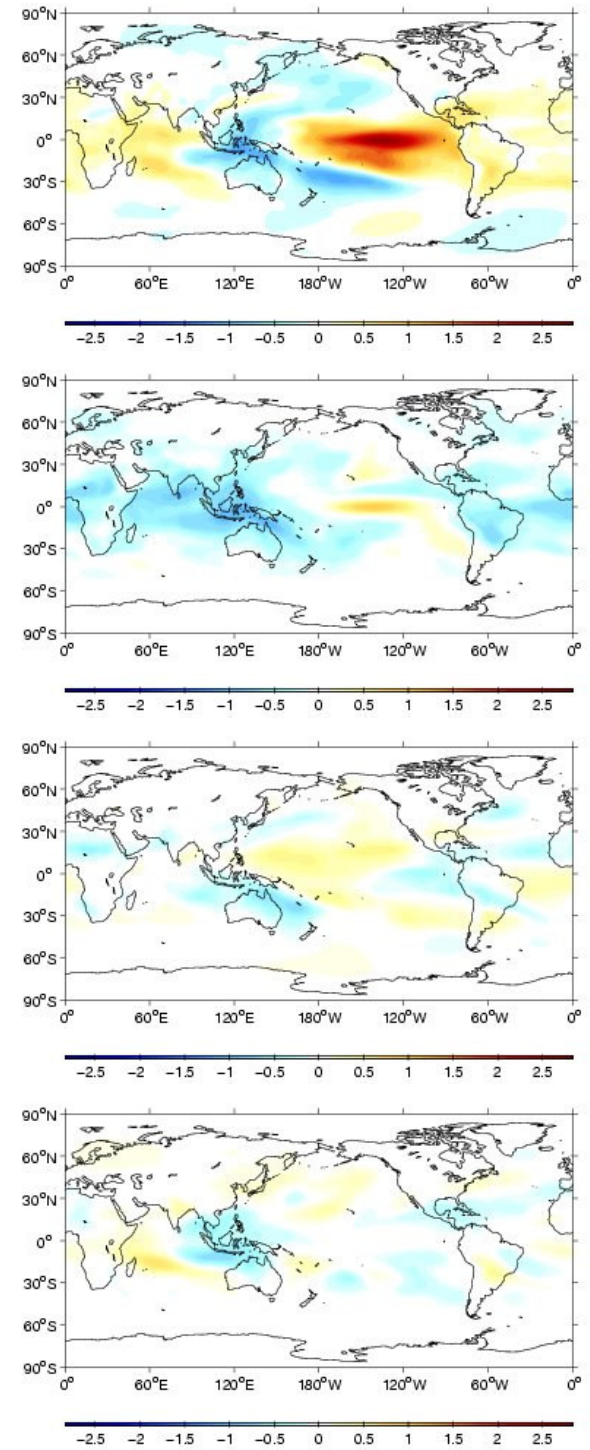

Fig. 6. The spatial patterns of the four leading interannual components extracted from the combined data.

prediction of future data and as the first, exploratory stage of interpreting the data. A data-driven approach cannot identify the underlying physical mechanisms of the found phenomenon but it can identify targets for future studies. For instance, with a low-pass filter, DSS extracts a very clear trend from the data. This may or may not be related to, say, global warming and more research is required to assess this, but DSS can certainly provide a good starting point for future research.

\section{ACKNOWLEDGEMENTS}

The authors would like to thank the NOAA-CIRES Climate Diagnostics Center, Boulder, Colorado, USA, for providing NCEP Reanalysis data from their Web site at http://www. cdc.noaa. gov, and Dr. Rich Pawlowicz for providing the mapping toolbox for Matlab.

\section{REFERENCES}

[1] M. H. Glantz, Currents of Change: El Niño's Impact on Climate and Society. Cambridge University Press, 1996.
[2] K. E. Trenberth, "The definition of El Niño," Bulletin of the American Meteorological Society, vol. 78, no. 12, pp. 2771-2777, December 1997.

[3] K. E. Trenberth and J. M. Caron, "The Southern Oscillation revisited: Sea level pressures, surface temperatures, and precipitation," Journal of Climate, vol. 13, pp. 4358-4365, December 2000.

[4] K. E. Trenberth, "Signal versus noise in the Southern Oscillation," Monthly Weather Review, vol. 112, pp. 326-332, 1984.

[5] J. Särelä and H. Valpola, "Denoising source separation," Journal of Machine Learning Research, vol. 6, pp. 233-272, 2005.

[6] A. Hyvärinen, J. Karhunen, and E. Oja, Independent Component Analysis. J. Wiley, 2001.

[7] E. Kalnay and Coauthors, "The NCEP/NCAR 40-year reanalysis project," Bulletin of the American Meteorological Society, vol. 77, pp. 437-471, 1996.

[8] K. E. Trenberth and D. P. Stepaniak, "Indices of El Niño evolution," Journal of Climate, vol. 14, pp. 1697-1701, April 2001.

[9] D. J. Karoly, "Southern Hemisphere circulation features associated with El Niño-Southern Oscillation events," Journal of Climate, vol. 2, pp. $1239-1252,1989$.

[10] H. Valpola and J. Karhunen, "An unsupervised ensemble learning method for nonlinear dynamic state-space models," Neural Computation, vol. 14, no. 11, pp. 2647-2692, 2002. 